\title{
ADOPCIÓN Y APLICACIÓN DE LAS NORMAS INTERNACIONALES DE CONTABILIDAD EN LAS PEQUEÑAS EMPRESAS, UCHUMAYO, AREQUIPA, 2013
}

\author{
ADOPTION AND IMPLEMENTATION OF \\ INTERNATIONAL ACCOUNTING STANDARDS IN SMALL \\ ENTERPRISES, UCHUMAYO, AREQUIPA, 2013.
}

Jhulyta Aguirre Cancino, Dennise M. Alvarez Ydme, Ivón R. Huacoto Huacollo, Carlos M. Neyra Olanda

Universidad Católica San Pablo, Arequipa, Perú

\section{Resumen:}

El presente estudio de investigación planteó como objetivo analizar si las Pequeñas Empresas adoptan y aplican las NIIF en Uchumayo, Arequipa. Para ello se elaboró y validó un cuestionario de nueve preguntas con índices aceptables de validez y confiabilidad que fue aplicado a 40 representantes o contadores de pequeñas empresas de dicho distrito. Para tal efecto, los resultados señalan que dichas entidades, si bien han comprendido la importancia, la relevancia de la adopción y aplicación de las NIIF, así como también han realizado inversión sobre todo en tecnología y de manera mínima en recursos humanos, también es verdad que la mayoría de estas entidades conocen muy poco sobre estas normas, lo cual refleja un inadecuado manejo y aplicación y, por ende, trae consigo un resultado moderado y no óptimo.

Palabras clave: Pequeñas empresas, NIIF, adopción.

\section{Summary:}

This present research study has posed as its main objective to analyze whether or not small enterprises are currently adopting and implementing IFRS in Uchumayo, Arequipa. For 
this specific purpose, a questionnaire containing nine questions all with satisfactory levels of validity and reliability was developed and applied to 40 representatives or small business accountants of said district. To this end, the results indicate that most of these entities, despite having understood the importance and relevance of the adoption and implementation of IFRS, and having made notable investments in technology and minimal investments in human resources, know very little about these rules and regulations. These findings reflect an inadequate handling (management) and application, thus, bringing about a moderate and non-optimal result.

Key Words: Small Enterprises, IFRS, adoption

\section{Introducción}

En las últimas décadas, mediante diversos pronunciamientos del área contable, así como de algunas normas legales, se estableció en Perú la obligatoriedad de preparar y presentar los estados financieros de las empresas del sector privado en base al modelo de las Normas Internacionales de Contabilidad (NIC), hoy Normas Internacionales de Información Financiera (NIIF), oficializadas en el país con el fin de lograr un proceso de armonización contable, que permita aplicar estándares internacionales. Pero, cabe preguntarse, ¿cómo se viene dando esa adopción y aplicación en las PYMES y, específicamente, en las Pequeñas Empresas?

Según la Ley $\mathrm{N}^{\circ}$ 28015, Ley de Promoción y Formalización de la Micro y Pequeña Empresa, define a las MYPES en su artículo segundo como una unidad económica constituida por una persona natural o jurídica, bajo cualquier forma de organización o gestión empresarial contemplada en la legislación vigente, que tiene por objeto desarrollar actividades de extracción, transformación, producción, comercialización de bienes o prestación de servicios (Ley de Promoción y Formalización de la MYPE, 20o8).

$\mathrm{Al}$ respecto, el Instituto Nacional de Estadística e Informática (INEI), en uno de sus reportes emitidos este año, indica que en Perú existen, al 30 de junio de 2013, 1 millón 713 mil 272 unidades empresariales, de las cuales el $96,2 \%$ son micro empresas, el $3.2 \%$ pequeñas empresas y el $0.2 \%$ medianas empresas, teniendo en cuenta que todo ello se ha realizado tomando en consideración la nueva categorización empresarial establecida por la Ley $\mathrm{N}^{\circ}$ 30056, "Ley que modifica diversas leyes para facilitar la inversión, impulsar el desarrollo productivo y el crecimiento empresarial", promulgada el 1 de julio de 2013, la cual, entre otros aspectos, elimina la cantidad de trabajadores que diferenciaba a las MYPES de la mediana empresa, siendo micro empresas las que alcanzan ventas anuales de hasta 150 UIT, las que superan este monto y hasta 1700 UIT es considerada pequeña, y medianas empresas las que alcanzan ventas anuales superiores al monto precedente y hasta 2300 UIT (Ley 30056, 2013). 
Ahora bien, y siguiendo la línea de lo señalado por el INEI, es importante destacar que Arequipa concentra el 5.6\% de cantidad de empresas, y sus ingresos por ventas es uno de los más altos a nivel nacional, donde las micro y pequeñas empresas son más importantes que las medianas y grandes empresas. Sin embargo, y a pesar de su incidencia en la economía de nuestra ciudad (y país) y la promoción para su desarrollo, crecimiento y formalización por parte del Estado, son todavía recurrentes diversos problemas, debido a las limitaciones que presentan en el área contable y, específicamente, en la adopción y aplicación de las NIIF por parte de las pequeñas empresas.

Las NIIF son normas emitidas por el International Accounting Standars Boards (IASB), organismo independiente del sector privado que desarrolla y aprueba las NIIF, y que basa su utilidad en la presentación de información consolidada en los Estados Financieros.

Con la adopción de las NIIF, desde 2005 la información financiera en el mundo ha experimentado una rápida evolución en sus estándares. Es así que se estableció en Perú la obligatoriedad de preparar y presentar los estados financieros de las empresas del sector privado en base al modelo de las NIC, hoy NIIF, oficializadas en el país; sin embargo, dichas normas no son aplicadas íntegramente en nuestro país (Guía Rápida de las NIIF2008), ya que cuando se prepara la información financiera se prioriza la norma tributaria frente a la norma contable (Becerra, 2010).

Es así que si bien el Consejo Normativo de Contabilidad (CNC), órgano encargado de la adopción y publicación de las NIIF en nuestro país, mediante Resolución 005-94EF/93.01 del 18 de abril de 1994, oficializó las NIIF para la profesión contable, y tres años más tarde, en 1997, la Ley General de Sociedades $\mathrm{N}^{\circ}$ 26887, en su artículo 223, estableció la obligatoriedad de que los estados financieros se preparen y presenten de conformidad con las normas legales y Principios de Contabilidad Generalmente Aceptados, práctica posteriormente aclarada por la Resolución 013-98-EF/93.01 del CNC en 1998, así como por la aprobación oficial de las NIIF para PYMES, cuyo uso obligatorio fue a partir del 1 de enero de 2011; se puede afirmar que sólo las empresas grandes y transnacionales han estado aplicando en forma completa los requerimientos de las NIIF vigentes por tener los recursos necesarios para poder cumplirlas, además de la responsabilidad pública de informar al mercado los resultados de sus operaciones y su situación financiera.

Las NIC o NIIF son una realidad, y un estudio elaborado por las siete más importantes firmas de auditoría dice que deben ser aplicadas en todo el mundo, sin embargo y a pesar de su aceptación, el camino no es fácil sobre todo en los países como el nuestro donde los cambios están costando, y más aún si se trata de una pequeña empresa.

Es así y conforme se ha indicado, que si bien las Pequeñas Empresas representan el 3.2\% y un generador de la economía muy importante, entonces cabe preguntarse si éstas están adoptando y aplicando de manera idónea las NIIF, y si realmente "conocen" su verdadera aplicación, ya que de no ser así, 
los estados financieros de estas empresas resultarían confusos, y por ende, se tomaría decisiones inadecuadas basadas en información que no es real.

Y es que, para que los inversionistas y otros usuarios de la información financiera puedan actuar con total transparencia y confiabilidad, se requiere que esta información sea preparada de acuerdo a un marco normativo internacional. El alto grado de globalización que han alcanzado los mercados financieros, incluido el peruano, nos obliga a contar con un marco normativo contable que esté de acuerdo con estándares internacionales, que tenga plena vigencia, sea estable en el tiempo y que a su vez se adapte a los nuevos requerimientos de la economía mundial, de tal forma que los inversionistas y cualquier usuario que requiera información financiera, pueda actuar con total transparencia y confiabilidad (Díaz, 2010).

Pero, el reclamo y promoción de un lenguaje estable universal ha estado a cargo de las grandes corporaciones internacionales, las que aspiraban contar con un cuerpo normativo común, único y homogéneo que les permitiera formular sus estados financieros, de tal manera que éstos fueran aceptados en todos los mercados financieros del mundo en los que operaban, sin que fuese necesaria las costosas adaptaciones y modificaciones contables (Guevara \& Cruz, 2010).

La obligación antes mencionada alcanza a todas las compañías, desde aquellas grandes corporaciones que cotizan en bolsa hasta las pequeñas empresas en Perú. Las empresas que no tienen la responsabilidad de presentar información financiera al mercado, en muchos casos no cumplen con muchas de las NIIF vigentes y sólo llevan su contabilidad para efectos de cumplir con sus obligaciones de reporte ante la SUNAT, y en algunos casos, ante bancos y otras entidades financieras, y ello generalmente sucede en las pequeñas empresas.

Y es que un tema de vital importancia para las pequeñas empresas, es el relativo a la información adecuada y sistematizada sobre diversos aspectos vinculados con el negocio empresarial, lo que en definitiva tendrá incidencia en la calidad, productividad y competitividad (Peña y Bastidas, 2004).

Pero, surgen algunas interrogantes en cuanto a este tipo de empresas: ¿Han comprendido el proceso y la importancia de la adopción y cumplimiento de la norma? ¿Conocen realmente la verdadera importancia en su aplicación o la perciben como un mero formalismo contable-administrativo? ¿Cuál es la inversión en tecnología y en formación del recurso humano?.

En 1995 se firmó un acuerdo entre el IASC, rebautizado en 2001 como IASB y la IOSCO, que consistía en un modelo normativo referencial de contabilidad internacional. Al respecto, existen ciertos estudios e investigaciones a nivel nacional e internacional que prevén las dificultades de la adopción y aplicación de dichas normas en las pequeñas empresas como es el caso de Venezuela. Así Guevara y Rangel (2010) señalan que:

a) las NIIF no han sido diseñadas originalmente para PYME's, b) que dicho marco ha sido concebido por y para gran- 
des empresas y corporaciones, y c) que ha sido motivado, fundamentalmente, para promover el ahorro de los altos costos de transformación y adaptación de los estados financieros de las grandes empresas [...] están ocasionando grandes limitaciones y dificultades a las PYME's, especialmente si nos referimos a los altos costos de implementación de dichas normas $[\ldots]$

...las limitaciones para la aplicación de las NIIF a las PYME's no son particularmente difíciles para las empresas venezolanas, ni siquiera son especialmente difíciles y costosas, sino que lo son para empresa mediana y pequeña en cada uno de los países del mundo donde han sido adoptadas tales normas desde el año 2000".

En realidad, los aspectos mencionados anteriormente son un claro ejemplo de que al respecto existe una problemática que no es solo interna sino también externa, donde las pequeñas empresas, en la mayoría de los casos, no cumplen con muchas de las NIIF vigentes y sólo llevan la contabilidad para efectos tributarios.

Si bien la adopción de los estándares internacionales de contabilidad es un proceso que trajo consigo un verdadero cambio en el sentido de revolucionar todo el entorno de las PYMES, y específicamente, de las pequeñas empresas; sin embargo, para la adopción y aplicación íntegra de las referidas normas se requiere de una madurez tecnológica, financiera y de gestión que las PYMES, y en particular las de Arequipa, no tienen; por tanto, merece de un especial tratamiento, ya que no cuentan con la masa de recursos necesaria para competir con grandes empresas en los mercados internacionales. Por tanto, mediante el presente estudio pretendemos probar todos los desafíos y dificultades hacia este tipo de empresas en la ciudad de Arequipa en cuanto a la incidencia de la adopción y aplicación de las NIIF, ligado sobre todo al tema de conocimiento de estas normas (Humire, 2008).

\section{Material y métodos}

Se trata de un estudio no experimental, transeccional descriptivo, en la medida en que se busca describir la adopción y aplicación de las NIIF en las Pequeñas Empresas en un determinado momento, esto es, el año 2013, determinando su incidencia y valoración en que se manifiestan las variables a investigar de manera individual.

\section{Muestra}

La muestra está constituida por 40 Pequeñas Empresas del distrito de Uchumayo, cuya selección fue extraída del INEI, cuyo nivel de significancia es del $99 \%$ y un error de estimación de $1 \%(\mathrm{p}<0.01)$. La muestra está constituida por 28 empresas de servicios y 12 empresas comerciales, habiéndose aplicado a los representantes o contadores de las empresas.

\section{Instrumentos}

Como método de investigación se utilizó la encuesta; como instrumento de investigación se diseñó y elaboró un cuestionario que recogió información relativa a la adopción y aplicación de las Normas Internacionales 
de Contabilidad en las Pequeñas Empresas de Uchumayo en el año 2013. El cuestionario constó de una ficha de identificación de la empresa y del informante, donde se especificó el cargo que ocupa en la entidad, así también consta de 9 preguntas cerradas, las cuales son dicotómicas y de varias alternativas, y a su vez precodificadas.

\section{Tabla 1.}

Preguntas del cuestionario de adopción y aplicación de las NIIF en las PYMES de Arequipa

1. ¿Cuál es el grado de conocimiento de las NIIF?

2. ¿Considera Ud. relevante la aplicación de las NIIF para pequeñas empresas?

3. ¿Aplica las NIIF en su empresa?

4. De aplicar las NIIF ¿hace cuánto tiempo lo hace?

5. ¿Cuál es el impacto de la aplicación?

6. ¿Cuál es el resultado de la aplicación?

7. ¿Ha invertido en tecnología?

8. De haber realizado inversión ¿en qué intervalo se encuentra?

9. ¿Ha invertido en recursos humanos?

\begin{tabular}{|l|c|c|c|c|c|c|c|c|c|}
\hline & 1 & 2 & 3 & 4 & 5 & 6 & 7 & 8 & 9 \\
\hline Media & 1.80 & 1.08 & 1.15 & 2.18 & 1.25 & 1.88 & 1.10 & 1.95 & 1.15 \\
Error típ. & 0.10 & 0.04 & 0.06 & 0.18 & 0.07 & 0.11 & 0.05 & 0.13 & 0.06 \\
Mediana & 2.00 & 1.00 & 1.00 & 2.00 & 1.00 & 2.00 & 1.00 & 2.00 & 1.00 \\
Moda & 2.00 & 1.00 & 1.00 & 1.00 & 1.00 & 2.00 & 1.00 & 1.00 & 1.00 \\
Desv.Est. & 0.61 & 0.27 & 0.36 & 1.13 & 0.44 & 0.72 & 0.30 & 0.81 & 0.36 \\
Varianza & 0.37 & 0.07 & 0.13 & 1.28 & 0.19 & 0.52 & 0.09 & 0.66 & 0.13 \\
Curtosis & -0.34 & 9.74 & 2.26 & -1.08 & -0.59 & -1.01 & 5.98 & -1.48 & 2.26 \\
Coef.Asim. & 0.12 & 3.35 & 2.04 & 0.54 & 1.20 & 0.19 & 2.77 & 0.09 & 2.04 \\
Rango & 2 & 1 & 1 & 3 & 1 & 2 & 1 & 2 & 1 \\
Mínimo & 1 & 1 & 1 & 1 & 1 & 1 & 1 & 1 & 1 \\
Máximo & 3 & 2 & 2 & 4 & 2 & 3 & 2 & 3 & 2 \\
Cuenta & 40 & 40 & 40 & 40 & 40 & 40 & 40 & 40 & 40 \\
\hline
\end{tabular}

Para efectos de la presente investigación se validó el cuestionario, haciendo uso de la estadística descriptiva, utilizando diagramas circulares y de barras, explicando de esta forma la importancia e impacto que las normas referidas generan en dichas empresas.

\section{Procedimientos}

En primer lugar, se solicitó el permiso respectivo a las empresas que conforman parte de nuestra muestra. Este permiso fue concedido respetando el tiempo máximo a entrevistar que fue aproximadamente de 5 minutos.

La recolección de la información se hizo a través del cuestionario de adopción y aplicación de las NIIF en las pequeñas empresas de Uchumayo, fue aplicado a los representantes o contadores en su horario de trabajo.

\section{Resultados}

En función de los resultados de las encuestas obtenidas, se procedió a aplicar a cada pregunta las medidas de tendencia central y de dispersión que se muestran líneas abajo.

\section{Tabla 2.}

Descriptivos de adopción de aplicación de las NIIF

¿Cuál es su grado de conocimiento de las NIIF's?

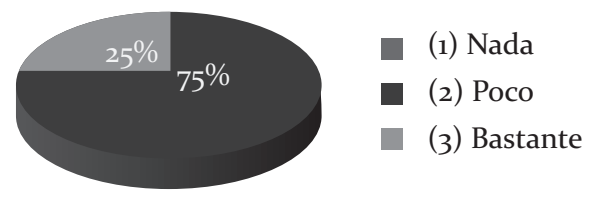


Por tanto, y teniendo en cuenta los resultados obtenidos respecto de las medidas respectivas, se tiene, en primer lugar, que en cuanto al grado de conocimiento de las NIIF prima su poco conocimiento por parte de las empresas encuestadas, lo cual se encuentra representado por el $75 \%$ de la muestra, muy por el contrario sólo el $25 \%$ de ellos indica conocer bastante sobre el tema.

Se puede observar también que la totalidad de las pequeñas empresas, representado por el $100 \%$, consideran de suma importancia su aplicación.

¿Considera Ud. relevante la aplicación de las NIIF para las pequeñas empresas?

(2) Sí

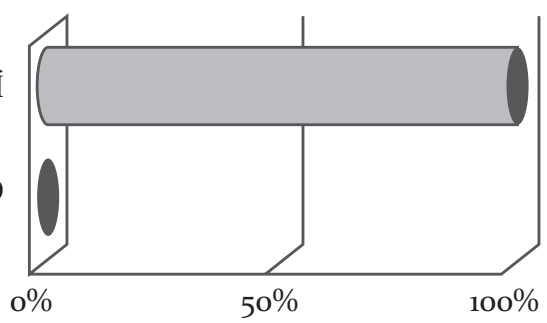

Por tanto, el $100 \%$ de ellas indica también aplicarlas en sus empresas.

¿Aplica las NIIF en su empresa?

(2) Sî

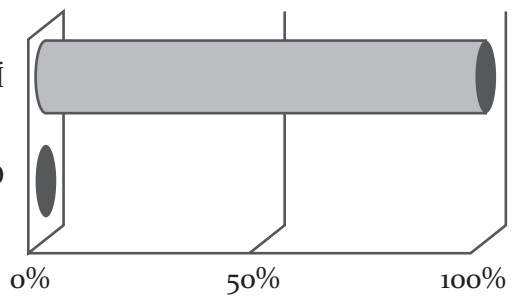

Así también, se puede observar que el $45 \%$ de las pequeñas empresas aplican las normas internacionales de contabilidad hace 2 años, mientras que un $35 \%$ viene aplicándolas hace 1 año y sólo un $20 \%$ las aplica hace 3 años.

¿De aplicar las NIIF hace cuánto tiempo lo hace?

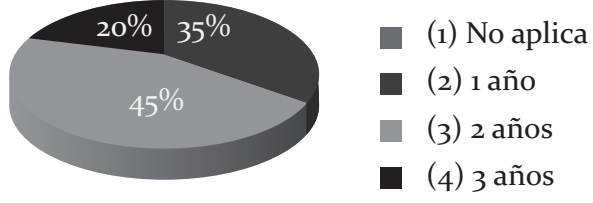

Por medio del siguiente diagrama se aprecia que el impacto de las empresas que vienen aplicando las normas es positivo, el mismo que está representado por la totalidad de las pequeñas empresas encuestadas, lo cual evidencia su importancia.

¿Cuál es el impacto de la aplicación?

(2) Sí

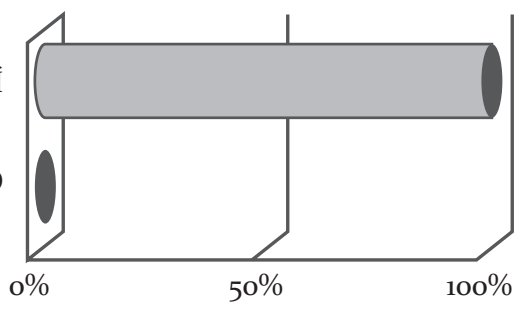

Ahora, si bien el impacto de la aplicación es muy positivo; sin embargo, su resultado no es óptimo, debido a que éste sólo se encuentra representado por un $20 \%$, tratándose más bien de un resultado moderado, el mismo que equivale a un $80 \%$. 
¿Cuál es el resultado de la aplicación?

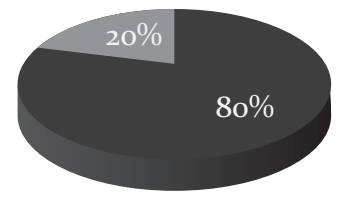

Todo lo cual ha generado que las empresas realicen inversión en tecnología, la que conforme se aprecia en el diagrama siguiente está representado por un $72.50 \%$ de las entidades encuestadas que han realizado este tipo de inversión, siendo aproximadamente el 30\% quienes no han invertido en este aspecto.

¿Ha invertido en tecnología?

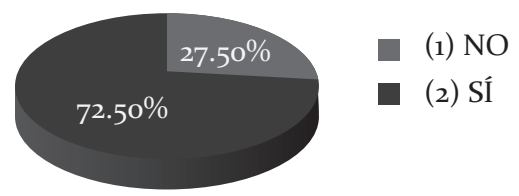

Es así que la inversión realizada en tecnología abarca un monto entre 3,000 a 10,000 nuevos soles, lo cual representa un $70 \%$, mientras que un $30 \%$ ha realizado una inversión inferior a 3,00o nuevos soles.

De haber realizado inversión ¿en qué intervalo se encuentra?

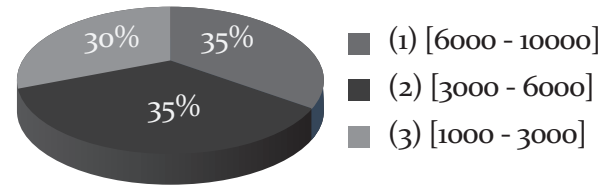

Muy por el contrario, sólo el 15\% de las entidades encuestadas han invertido en recursos humanos.

¿Ha invertido en recursos humanos?

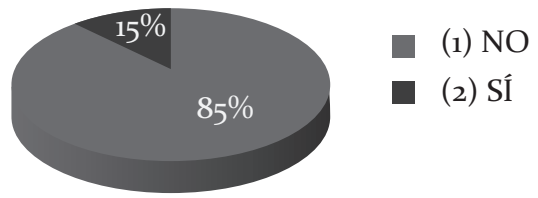

\section{Discusión}

La adopción y aplicación de las Normas Internacionales de Contabilidad son de suma importancia debido al carácter universal que éstas tienen en el mundo de la contabilidad; sin embargo, se ha podido presentar que lamentablemente su adopción y aplicación tiene un carácter particular.

En primer lugar, se ha podido demostrar que en la totalidad de empresas encuestadas, éstas vienen aplicando dichas normas con un período de antigüedad de uno a tres años aproximadamente, teniendo en cuenta que un $35 \%$, cifra considerable, lo viene realizando hace un año, lo cual es un aspecto favorable; además se ha demostrado que su aplicación tiene un impacto muy positivo, considerándose además relevante su aplicación y generándose una inversión en tecnología, sobre todo, entre 3,000 y 10,000 nuevos soles aproximadamente.

El problema y la interrogante surge en cuanto al grado de conocimiento que indican tener los especialistas en la materia, así como los representantes de las pequeñas empresas, quienes han indicado "saber poco" acerca de las Normas Internacionales de Información Financiera, cuyo resultado de aplicación es moderado, el cual se encuentra reflejado por el $80 \%$, mientras que 
sólo el $20 \%$ indica que se ha generado un resultado óptimo.

Por tanto, al existir un conocimiento parcial, es poco probable que las pequeñas empresas, quienes están obligadas a utilizar estas normas y conforme a la regulación existente, apliquen de manera adecuada las NIIF, lo que conlleva a que los estados financieros de estas empresas se tornen confusos y por ende se tomen decisiones inadecuadas, ya que están basadas en información que no es real, más aún que estas entidades brindan información anual a la SUNAT y no cada tres meses como sí lo hacen las empresas que cotizan en Bolsa, generándose de esa forma un cierto vacío en el sentido de que no existe ningún organismo que los controle para su correcta aplicación.

Es así que se comprueba la hipótesis planteada al determinar que, efectivamente, en Arequipa las Pequeñas Empresas no aplican de manera idónea y bajo los parámetros establecidos las NIIF, lo cual evidentemente perjudica en la efectiva toma de decisiones en el ámbito empresarial.

Su aplicación es necesaria e importante, debido a que hoy en día las empresas se desarrollan dentro de un mundo globalizado y fuertemente competitivo; sin embargo, se debe prever las medidas necesarias a efecto de que las PYMES y especialmente las Pequeñas Empresas puedan tener acceso a este tipo de conocimiento y por ende realizar su respectiva aplicación, ya que ello les brindará una mayor sofisticación en diversos aspectos.

Se ha citado que una de las dificultades y desafíos es el costo en la implementación de la tecnología y recursos humanos, ya que dichos montos muchas veces no podrán ser afrontados por las referidas empresas, al requerirse de sistemas informáticos más personalizados que desde luego algunas pequeñas empresas no podrán afrontar, teniendo en cuenta sus ingresos percibidos anualmente, para ello es necesario que se adopten otro tipo de sistemas que se adecúen a este tipo de empresas y permitan formalizarlos de manera más apropiada.

Por tanto, es imprescindible que se apliquen las NIIF en las PYMES, pero tomando en consideración todos los aspectos que involucran y que, sobre todo, están relacionados con el factor contable, administrativo y financiero, a efecto de que aquellas que vienen implementando este sistema puedan verse aún más beneficiadas y pasen a un nivel óptimo de aplicación, requiriéndose de un cambio en la estructura de la empresa, lo cual implica un proceso que conlleve una adecuada capacitación por parte de los especialistas, porque ello ofrece muchas ventajas y permite a las empresas ser más competitivas.

Para finalizar, agradecemos a los miembros de las pequeñas empresas encuestadas por su gentil colaboración. 


\section{Referencias}

Amat, O. (2004). Comprender las normas internacionales de contabilidad NIC. Barcelona: Gestión.

Andrade, S. (2002). Normas internacionales de contabilidad Perú: Editorial y Librería Lucero SRL.

Arroyo, A. (2011). Normas internacionales de información financiera y el peritaje contable. "Contabilidad y Negocios", 6, 67-81. Ayala, P. (2011). Aplicación práctica de las Normas Internacionales de Información Financiera (NIIF, NIC, CINIIF y SIC) Perú: Pacífico Editores SAC.

Ayala, P. (2013). NIIF comentarios y casuística Perú: Instituto Pacifico SAC.

Carrillo, M. (1999). La contabilidad de costos y la adopción en Venezuela de las Normas Internaciones de Información Financiera”. "Actualidad Contable" FACES, 21, 90-115.

Copa, T. y Gonzales, G. (2010). Atractivo financiamiento a las pequeñas y microempresas para BCP, 3, 6o-8o. Esan Ediciones.

Díaz, O. (2010). Estado actual de la aplicación de las NIIF en la preparación de estados financieros en las empresas peruanas. "Contabilidad y Negocios", 5, 5-28.

Díaz, O. (2010). Panorama actual del marco normativo contable. "Informe Contable", 3, 9-10.

Estudio Caballero Bustamante. (2003). Normas internacionales de contabilidad Perú: Estudio Caballero Bustamante.

Federación de Colegios de Contadores Públicos, (2004). Normas internacionales de contabilidad. Perú: Contadores Públicos del Perú.
García, J. (2012). NIIF para PYMES Perú: Pacífico Editores SAC.

Gómez, M. (2004). Una evaluación del enfoque de NIIF desde la teoría de la contabilidad y control. "Innovar", 14, 112-131.

Guevara, I. y Rangel, P. (2010). Reflexiones sobre la aplicación de las NIIF en las PYMES Venezolanas. "Compendium", $13(25), 23-41$.

Humire, G. (2008). Necesidad de una norma internacional de información financiera para PYMES en el Perú. "Contabilidad y Negocios", 3, 05-10.

IFRS. (2012). Normas Internacionales de Información Financiera, United Kingdom: IFRS Foundation.

Lam, A. (2007). Convergencia y armonización de la normativa contable: hacia la contabilidad internacional. "Contabilidad y Negocios", 2, 6-15.

Loayza, C. (2000). Normas Internacionales de Contabilidad, Perú: Pacífico Editores SAC.

Márquez, Y. (2006). El sistema nacional de contabilidad en el Perú. "Contabilidad y Negocios", 1, 26-29.

Miranda, J. (2006). Hacia una necesaria explicación de la normatividad contable en el Perú, ¿Principios de Contabilidad Generalmente Aceptados?. "Contabilidad y Negocios", 1, 4-7.

Palacios, M. y Martínez, I. (2005). El proceso de armonización contable en Latinoamérica: camino hacia las normas internacionales. "Contabilidad y Finanzas", $16,103-117$

Paredes, C. (2009). Normas internacionales de contabilidad: Normas internacionales de información financiera. Interpretaciones SIC y NIIF Perú: Instituto Pacifico. 
Pereda, J. (2012). Contabilidad y desarrollo económico. "Contabilidad y Negocios", 7, 94-110.

Rodríguez, J. (2009). Adopción por primera vez de las NIIF. Un análisis teórico de la norma. "Contabilidad y Negocios", 12, 84-99.
Valdivia, C. (2002). Normas Internacionales de Contabilidad NICS e interpretaciones Perú: Pacífico Editores SAC.

Vílchez, P. (2008). La armonización de normas contables en los países de América. "Contabilidad y Negocios", 3, 5-10. 